\title{
BODIES IN FLOWING PLASMAS: LABORATORY STUDIES
}

\author{
N. H. Stone* and U. Samir** \\ *Space Sciences Laboratory, NASA/Marshall Space Flight \\ Center, Huntsville, Alabama, USA \\ **Department of Atmospheric and Oceanic Science, University \\ of Michigan, Ann Arbor, Michigan, USA
}

\section{ABSTRACT}

A brief review of early laboratory investigations of bodies in flowing, rarefied plasmas is given together with a discussion of more recent parametric studies carried out at NASA/Marshall Space Flight Center (MSFC), which include the effects of the ion acoustic Mach number and the normalized test body potential. Good agreement is found between the experimental results and theoretical calculations which omit ion thermal motion. The relation between laboratory investigations and the results of satellite-borne measurements is addressed. This relationship has led to an appreciation for the benefits of applying the methods and techniques of laboratory plasma physics to investigations in space, where several 1imitations inherent to the laboratory can be circumented. These types of investigations, conducted in Earth orbit, can enhance our understanding of space plasma physics and have direct application to certain types of solar system phenomena.

\section{INTRODUCTION}

One of the most frequently occurring phenomena in space plasma physics is the interaction which occurs between the tenuous, streaming space plasma and various types of celestial bodies. With the advent of the satelitte era, in situr measurements of the nearest and most important such phenomena, the Earth's magnetosphere, became possible along with studies of a more technical but related phenomenon, the interaction between the artifictal satellites themselves and the ionospheric plasma [1]. In more recent years, planetary probes have made possible important, albeit fragmentary, observations of additiona1 examples of the solar wind's interaction with other bodies of the solar system (1.e., the planets Mercury, Venus, Mars, and Juptter, and Earth's moon) and the interaction of Io with the Jovian magnetospheric plasma.

Long before the satellite era, however, some very rudimentary studies were carried out in the laboratory which revealed phenomena that have subsequently been observed in space. For example, experiments conducted by Birkeland in 1908 resulted in the discovery of field-aligned currents which now bear his name [2].

A more sophisticated series of experiments, conducted by Danielsson and Lindberg $[3,4]$ in the mld-1960's, confirmed the key features of the interaction between the interplanetary and geomagnetic fields predicted earlier by Dungey [5], before these effects were observed in space (i.e., a closed magnetic cavity for aligned fields 
and an open cavity containing a reversed plasma flow, for opposed fields). These and other examples are discussed in more detail in a review paper by Falthammar [6]. We should also mention more recent studies by Dubinin, et a1. [7], Podgorny and Andrijanov [8], and Podgorny [9].

The application of laboratory studies to space plasma phenomena is usually made through the concept of qualitative scaling. This concept is directed toward reproducing the physical mechanisms involved in a phenomenon rather than its exact morphology. As a result, it is required only that, if a quantity in space is much greater or much less than unity, then the inequality must be reproduced by the quantity in the scaled experiment but not necessarily to the same order of magnitude. Only when the quantity in space is on the order of unity must its value be closely preserved in the scaled experiment. This greatly relieves the stringent requirements imposed by classical Vlasov scaling. Unfortunately, even with these relaxed requirements, compromises must be made when scaling large solar system phenomena for study in the laboratory. For example, the requirements of an embedded magnetic field and an ion Larmor radius much smaller than the obstacle necessitate a rather high density which results in collision-dominated processes rather than the collisionless processes found in space. In addition, boundary layers and magnetic cavities are too small for detailed probing. Therefore, although some very significant results have been obtained, it appears that the investigation of large-scale phenomena in the laboratory suffers from several inherent limitations.

In the case of the interaction of artificial satellites with the ionosphere, the scaling requirements are far less demanding, and consequently, collisionless flow interactions can be maintained. Although this class of experiments also has limitations such as wall effects and non-thermalized particle distributions, a large number of studies have been carried out which have provided a relatively complete picture of the electrostatic plasma flow interaction (i.e,, omitting the embedded magnetic field).

In this paper, we will review very briefly some of the major findings and discuss in more detall a few particular results that have significantly influenced our understanding of the applicable in situ data from ionospheric satellites. Finally, we will explore some of the possibilities of exploiting the ionosphere as a laboratory with a natural and unbounded plasma in which certain types of solar system plasma phenomena can be studied more effectively.

\section{RESULTS FROM LABORATORY EXPERIMENTS}

Bodies in Cold-Nommagnetized Flowing Plasma

Typically this type of study has been conducted in steady plasma wind tunnel facilities. These facilities have been adequately described elsewhere [10,11,12] and will not be discussed here, except to mention the range of parameters available in the MSFC facility, given in Table 1. These values are fairly typical and indicate the degree of ionospheric simulation possible. The main limitations Involve the lack of an embedded magnetic field and Ion temperature, which will be discussed later.

One of the earliest studies was carried out by Ha11, Kemp, and Sellen in 1964 [13]. Although the electron temperature apparently was not measured (and, thus, the dimensionless parameters are not available for this work), for a negatively biased test body, they observed a strong ion density enhancement in the center of the ion void created downstream from the body. This effect is shown by the data presented in Figure 1. These profiles or normalized ion current density, $\mathrm{J} / \mathrm{J}_{0}$, were obtained in the MSFC facility for the conditions: 
Bodies in Flowing l'lasma: Laboratory Scudies

TABLE I Plasma Properties and Scaling Parameters

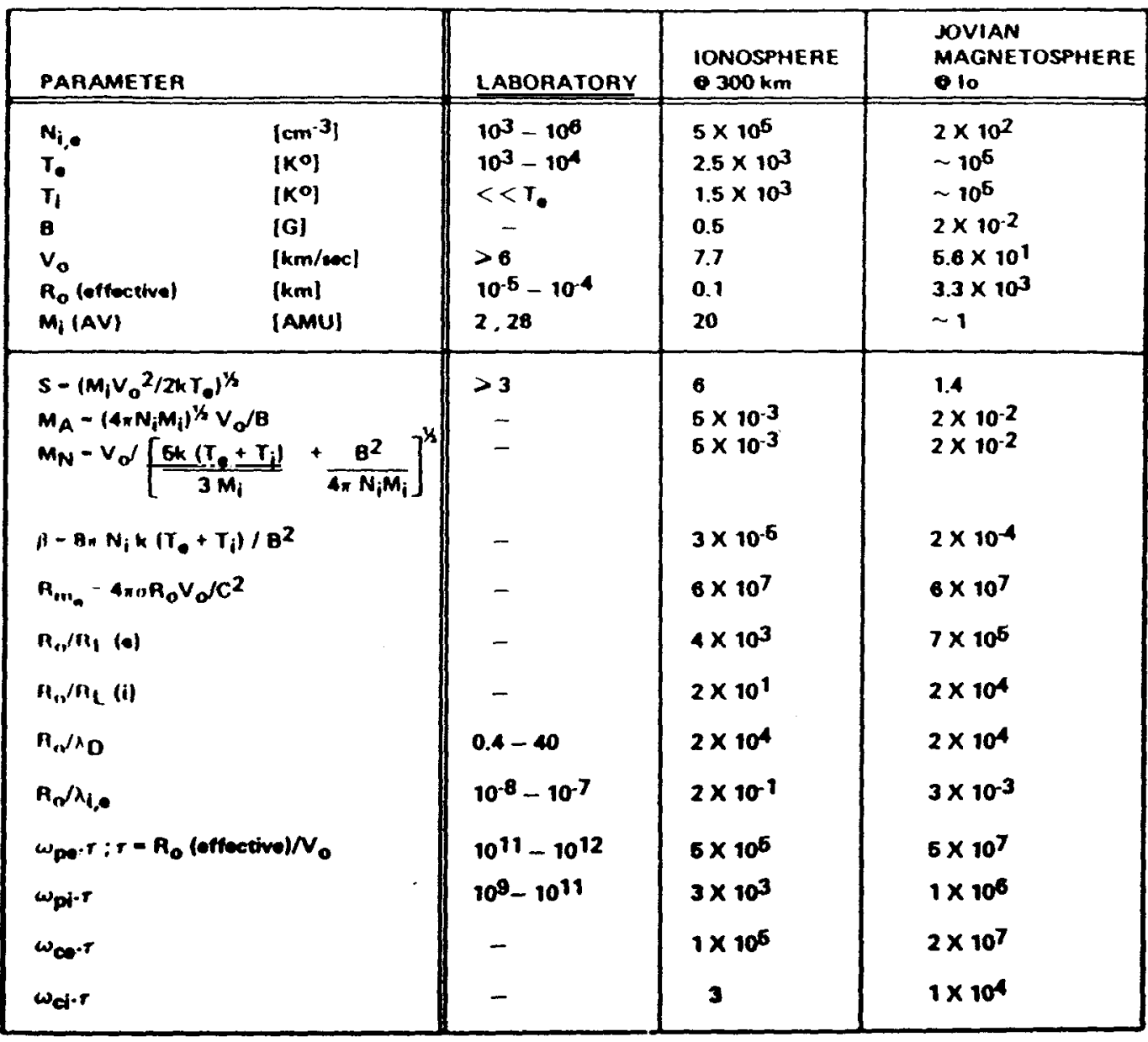

$$
\mathrm{S} \equiv\left(\mathrm{m}_{\mathrm{i}} \mathrm{V}_{\mathrm{o}}^{2} / 2 \mathrm{kT} \mathrm{e}^{\frac{1}{2}}=16.5, \mathrm{R}_{\mathrm{d}} \equiv\left(\mathrm{R}_{\mathrm{o}} / \lambda_{\mathrm{D}}\right) \simeq 1 \text {, and } \Phi_{\mathrm{b}} \equiv\left(\mathrm{e} \phi_{\mathrm{b}} / \mathrm{kT} \mathrm{e}_{\mathrm{e}}\right)=-5\right. \text {, }
$$

where $V_{o}$ is the Ion drift velocity, $R_{0}$ is the body radius, $\lambda_{D}$ is the Debye length, $\phi_{b}$ is the potential of the body relative to plasma potential, and the remaining symbols have the usual meanings. A year later, Clayden and Hurdle published more detailed results which showed the axial ion peak to occur for both spherical and conical bodies and indicated the lon current collected by the test bodies to depend on $\phi_{b} / s^{2}[14]$. A more recent investigation by Stone, et al., has demonstrated the plasma sheath thickness for spherical and cylindrical bodies to vary with $\left|\phi_{b}\right|^{\frac{1}{2}} / \mathrm{s}[15]$. . Since the collected current should depend on the effective crosssectional area of the test body and this increases as the square of the radius, these two results are in good agreement.

The existence of the axial ion peak in the mid-wake region of conducting bodies with $R_{d} \leq 40$ has subsequently been verified by a number of investigators. By compiling these results, it was found that the location of the peak for floating test bodies is given by $S R_{0}[16]$, as shown in Figure 2 . 


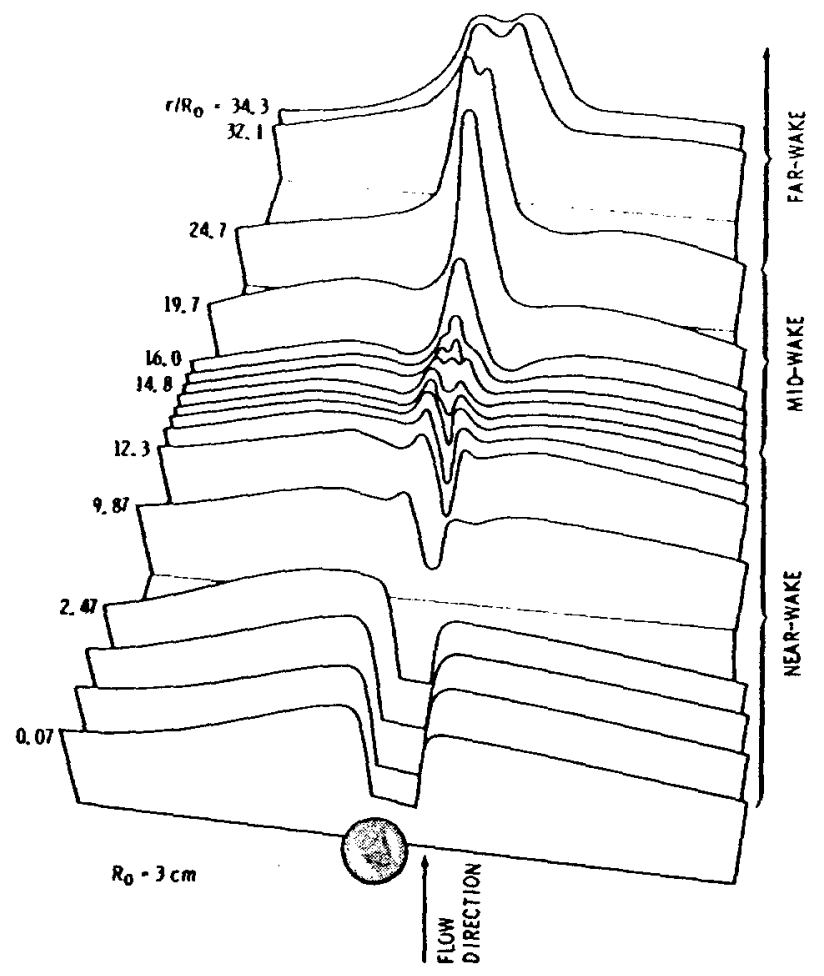

Fig. 1. Normalized ion current density profiles downstream from a conducting sphere. After [23]

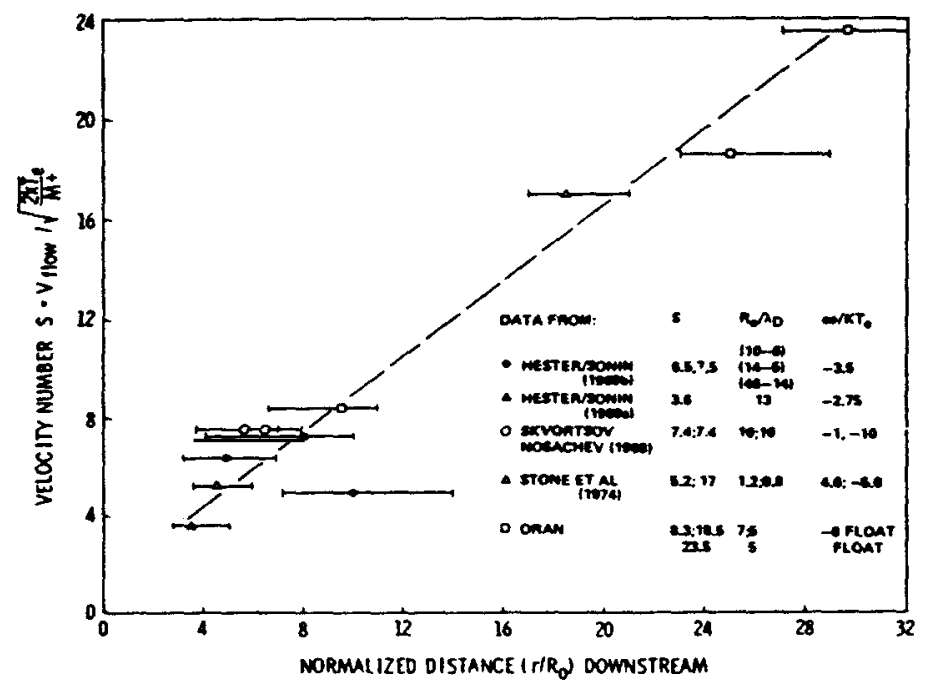

Fig. 2 The location of maximum ion current enhancement on the wake axis of axisymetric test bodies as a function of $S$. After [16] 
In 1968, Skortsov and Nosachev found a rarefaction wave to exist, creating a conical region of plasma depletion around the axial ion peak [17]. They also found a region of positive space potential to be associated with the axial ion peak. Finally, they demonstrated that the character of the axial ion peak is not preserved by scaling the ratio $\Phi_{b} / S^{2}$, but rhat $S$ and $\Phi_{b}$ must be preserved independently [18]. In agreement with this, it has subsequently been found that, for $\mathrm{R}_{\mathrm{d}} \approx 1$, the height and width of the axial ion peak depend on $\left(\mathrm{S} /\left|\Phi_{\mathrm{b}}\right|\right)^{\frac{1}{2}}$ and $\left|\Phi_{\mathrm{b}}\right|^{-\frac{1}{2}}$, respectively $[19]$. Additional peaks and diverging Mach cone type structures were found by Hester and Sonin to occur farther downstream from sma11 cylindrical bodies at intervals which depend on $\omega_{\mathrm{pi}} z / v_{0}$, where $\omega_{\mathrm{p} i}$ is the ion plasma frequency and $z$ is the wake axis $[20]$.

Referring to Figure 1 again, converging structures are clearly evident near the boundaries of the ion void in the near-wake region. Apparently these structures contribute to the ion void filling process as well as the creation of the axial ion peak in the mid-wake region. The existence of ion beams, created and focused onto the wake axis by the electric field in the plasma sheath, was predicted theoretically by Martin [21]. He also predicted that the angle of inclination of the beams to the wake axis should depend approximately linearly on $\Phi_{b}$. That the converging structures seen in Figure 2 are, in fact, the predicted ion beams is demonstrated in Figures 3 and 4. The data points in Figure 3 were obtained from several sets of ion current profiles obtained under the same conditions as the profiles of Figure 2 except that $\Phi_{\mathrm{b}}$ was varied as indicated. These data show that the angle of inclination to the wake axis, $\theta$, of both the converging ion beams and the ion void boundary, depend linearly on $\Phi_{b}$, while the diverging structure in the

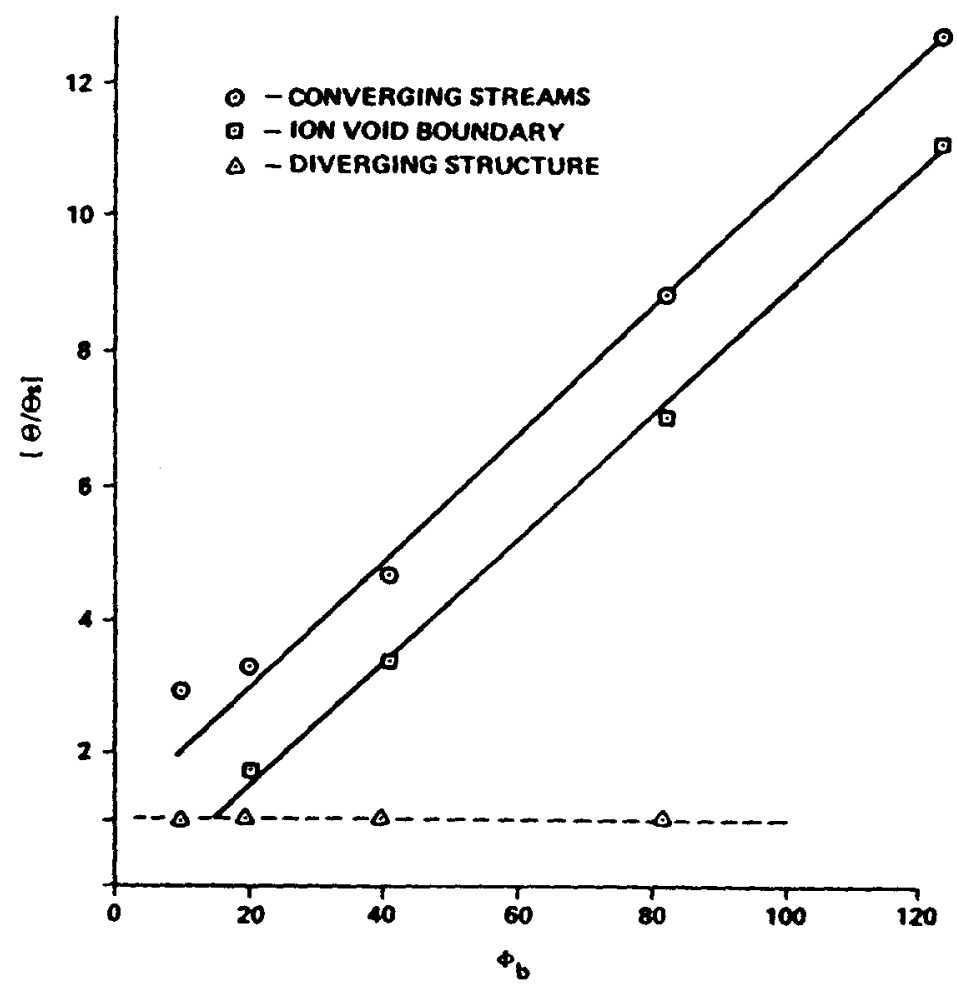

Fig. 3 The inclination of wake structures as a function of $\Phi_{b}$. After [23] 


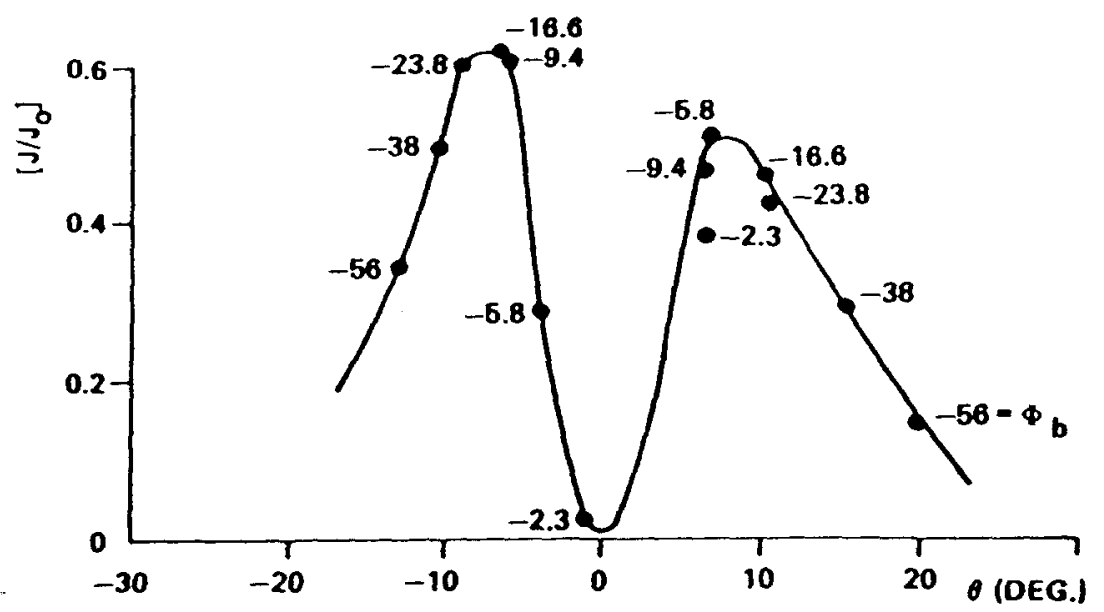

Fig. 4 Ion current density and inclination to the wake axis at $2 / R_{0}=$ 11.2 as a function of $\Phi_{b}$. After [19]

far wake is independent of $\Phi_{b}$ and, for this case, is inclined at the Mach angle, $\theta_{\mathrm{S}}=\sin ^{-1}(1 / \mathrm{S})$. The $\Phi_{\mathrm{b}}$-dependence would indicate that the converging structures are composed of ion streams; however, this is demonstrated more conclusively by Figure 4. These data points were obtained by differentially measuring the magnitude and direction (complete vector) of the ion flux [22]. The measurements were made at a fixed position, $2 / R_{0}=11.2$, on the wake axis while changing the test body potential. Note that at each value of $\Phi_{b}$, two ion streams were observed, one arriving from the left and one from the right side of the test body. Further, the envelope of the data points indicates the shape of the converging ion beams, which were deflected past the diagnostic instrument as $\Phi_{b}$ was made more negative [19]. The position at which the converging beams intersect the wake axis has been found to vary linearly with $S$, in agreement with the peak position shown in Figure 2 , and to depend on $\left|\Phi_{b}\right|^{-\frac{1}{2}}[23]$. The $\Phi_{b}$ dependence was predicted by Martin [21] and is demonstrated by experimental data in Figure 5. We have, therefore, demonstrated that the crossing point of the converging ion beams on the wake axis, $Z / R_{0}$, depends linearly on $\mathrm{S} /\left|\Phi_{\mathrm{b}}\right|^{\frac{1}{2}}$. This result will be found useful in analyzing satellite data in the following section.

\section{Effects of Ion Thermal Motion}

The most extensive work on the effects of cold ions in collisionless plasma wind tunnels has been done by Fournier and Pigache [24]. They have demonstrated, in agreement with several theoretical predictions $[25,26,27]$, that the general effect of ion thermal motion is to diffuse the detailed wake structure discussed above and that if $T_{e} / T_{i}$ becomes less than unity, the structure vanishes. However, the more quantitative question as to what value of $T_{e} / T_{i}$ causes the structures to vanish and whether this would be the case in the ionosphere, where $T_{e} / T_{i} \cong 2$ can occur, has not been satisfactorily resolved. In the following section we will consider data that suggest that the wake does, in fact, have structures in the ionosphere.

\section{Effects of an Embedded Magnetic Field}

Experimental work with magnetized plasma streams has been reported by Bogashchenko, et al., [28] and Astrelin, et al., [29]. These studies deal with the frequency and 


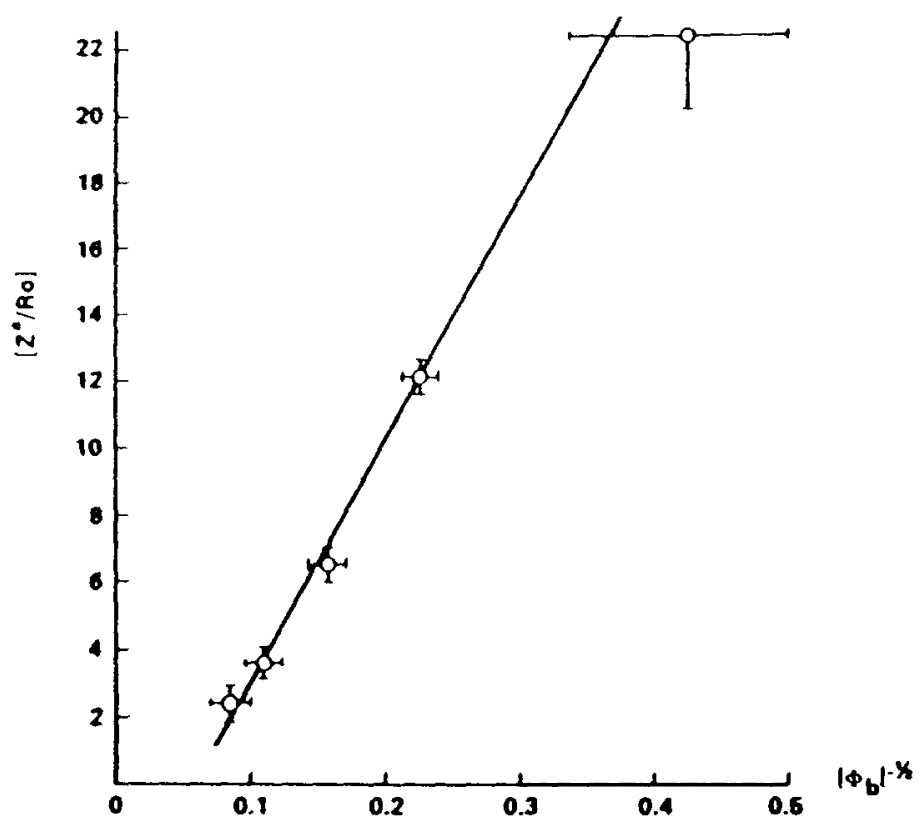

Fig. 5 Location of the intercept of the converging ion streams with the wake axis as a function of $\left|\Phi_{b}\right|^{-\frac{1}{2}}$. After [23]

amplitude of an oscillatory structure produced in the wake by ion cyclotron motion. However, when scaled to ionospheric conditions, the results of these studies indicate that the first enhancement of this type would occur ${ }^{200} \mathrm{~m}$ downstream and would be relatively small in amplitude [19]. Therefore, it is not expected that the near- and mid-wake regions of ionospheric satellites are affected significantly by the geomagnetic field.

\section{SOME IMPLICATIONS OF LABORATORY RESULTS ON SATELLITE DATA}

There are a number of examples in which in situ data on the interaction between satellites and the ionospheric plasma have been elucidated by the results of laboratory investigations. Here we consider two examples which make use of the results given in the previous section.

\section{Evidence for a Mid-Wake Axial Ion Peak}

Figure 6 shows the normalized electron current collected by the Ariel 1 boom probe as a function of angle of attach at $x=5 R_{\text {Ariel }}$ from the spin axis. As a result of spin axis precession, the probe passed through the disturbance created by various parts of the satellite $[30]$. Curve (a) shows the wake of the spherical lon probe, and curve (b) shows that of the main body. In light of the preceding laboratory results, we note that: (1) the electron enhancement is observed at a Mach number of radii ( $S R_{0}$ ) downstream in the Ariel 1 wake, which is where the laboratory data predict the peak value of the enhancement to occur; (2) the enhancement is less than the ambient density, which is in agreement with the predicted effects of ion thermal motion; (3) if we can apply the simple relation found for the plasma sheath expansion, $\left|\Phi_{b}\right|^{\frac{1}{2}} / S$, we find the effective radius of the ion probe (which was a factor of six smaller in radius but biased six volts negative with respect to the satellite main body) to be approximately equal to that of the main body, which 


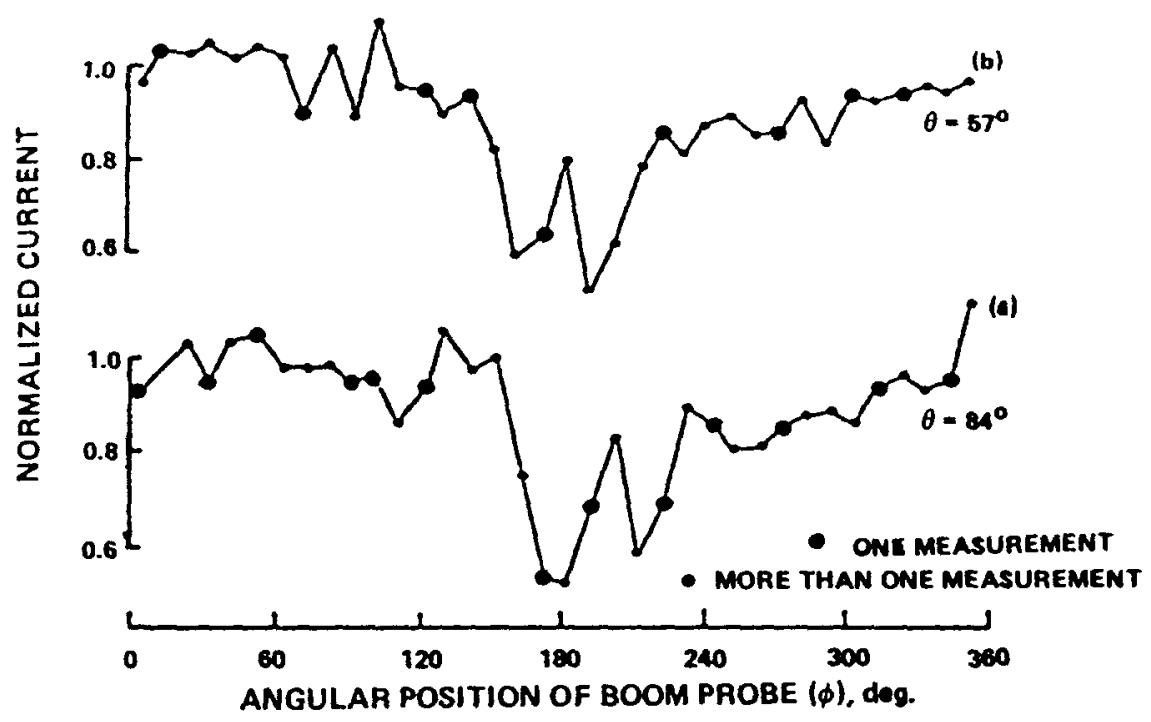

Fig. 6 Normalized electron current as a function of angle of attack for the case when the probe passes through the wake of (a) the Ariel I main body and (b) the spherical ion probe. After [30]

accounts for the similarity in the width of the two wakes, as well as the similarity in the positions of the axial electron enhancements (which actually occurred 33 radii downstream for the fon probe); and (4) it is to be expected that an electron enhancement would occur and exhibit characteristics similar to those of the ion enhancement as a result of laboratory observations [31].

\section{Ion Current Density in the Near-Wake Region}

The behavior of the ion current collected by a short, cylindrical Langmuir probe located 1.5 radil from the spin axis of the $\mathrm{AE}-\mathrm{C}$ satellite has been studied by Samir, et a1., [32]. In the referenced work, it was found that the normalized ion current collected in the satellite wake, $I_{w} / I_{0}$, depended on both the ratio of oxygen to hydrogen ion concentration, $n_{0} / n_{H}$, and the electron temperature. Here we extend this analysis by considering these data in light of the above laboratory results, which show the ions to be deflected by the plasma sheath and focused onto the wake axis, intercepting it at a position, $Z * / R_{0}$, which is proportional to $\mathrm{s} /\left|\Phi_{\mathrm{b}}\right|^{\frac{1}{2}}$.

Although $R_{d} \gg 1$ for $A E-C$ (and, hence, the sheath effects should be minor), while the laboratory results were obtained for $R_{d} \sim 1$, we can argue that the fon trajectories will be significantly affected by the negative space potential in the lon void region as they travel parallel to its boundary. If so, the parametric dependence of the two cases should be similar. Notice that, in Figure 1, the fon current density drops off monotonically along the wake axis upstream from the intercept of the converging ion streams. It is expected that this gradient in density would be spread even farther upstream by ion thermal motion in the ionospheric plasma.

Therefore, as $Z^{*} / R_{0}$ moves upstream (decreases), the ion current density should increase and we expect the normalized ion current measured by Samir, et aI., $I_{w} / I_{0}$, to be proportional to $\left|\Phi_{\mathrm{b}}\right|^{\frac{1}{2}} / \mathrm{s}$.

Note that $\Phi_{b}$ is no longer the satellite potential (which may be affected by imperfect conductivity and potential sources such as solar panels), but the negative 
space potential in the ion void region. We assume this to be proportional to the floating potential which we cilculate for a perfectly conducting sphere, using the measured electron temperature and the otbital velocity; i.e.,

$$
\Phi_{\mathrm{f}}=\frac{k T_{\mathrm{e}}}{\mathrm{e}} \ln \left[\left(\frac{\pi \mathrm{m}_{\mathrm{e}}}{8 \mathrm{kT}}\right)^{\mathrm{i} / 2} \mathrm{v}_{\mathrm{e}}\right]
$$

Assuming the ion species to act independently, we calculate the Mach number, $S_{i}$, for each species and multiply the ratio $\left|\Phi_{f}\right| 1 / 2 / s_{i}$ by the ratio of the ion ${ }^{\prime} s$ concentration to the total concentration, $n_{i} / n_{e}$. Adding both contributions, we have:

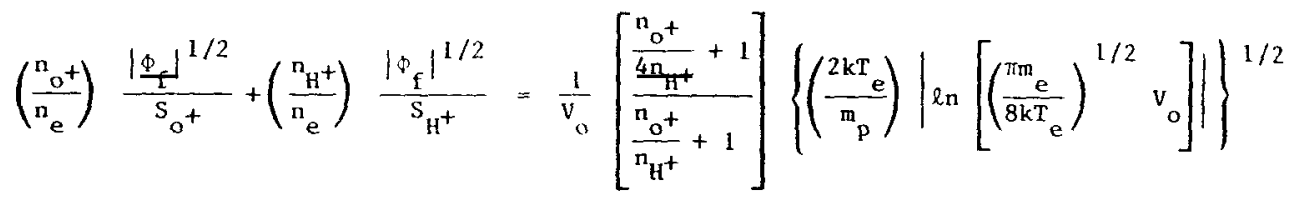

which is plotted against $I_{W} / I_{0}$ in Figure 7 . The fit obtained here, assuming independently acting ion constituents, is significantly bettex than that obtained using an average mass. This also shows that for this case, even though the scale size is vastly different from the laboratory, the influx of ions into the void region is governed by a similar parametric dependence.

\section{THE IONOSPHERE AS A NEW RAREFIED PLASMA LABORATORY}

The ionospheric plasma allows several limitations inherent to laboratory studies to be circumvented and, in addition, provides a new region of parameter space previously unavailable. Consider, for example, that the plasma is collisionless on a large scale. It, therefore, allows experiments to be conducted with correspondingly large-scale test bodies while the plasma flow interaction processes remain collisionless. The ionospheric plasma, for all practical purposes, is boundless and therefore not subject to perturbations created by walls or plasma confinement, and it is essentialiy isotropic with Maxwellian particle distributions. Depending on orbital inclination and altitude, steady conditions may exist for several minutes and the plasma composition can range from essentially pure oxygen to pure hydrogen ions. In terms of parameter space, in addition to large scale sizes, the lonosphere offers a collisionless, supersonic-subAlfvenic plasma which is difficult, if not impossible, to achieve in the laboratory.

We discuss here the application of the ionospheric laboratory to basic space plasma physics and illustrate, with a specific example, its application to some types of solar system plasma phenomena.

\section{Application to Space Plasma Physics}

The large range of scale sizes available in the ionosphere permits a comparative investigation of the fluid and kinetic formulations of the theory. The Debye length and Larmor radius may form effective Knudsen numbers with the available scale size range, including the transition region where the classical knudsen concept does not clearly indicate the validity of a fluid approach. Further, if a sufficiently strong magnetic dipole can be generated within the test body, the interaction of the dipole and geomagnetic fields and the resulting magnetic cavity can be studied. With such large scale sizes, the magnetic cavity may be sufficiently large to permit measurements of the induced current system within it. 


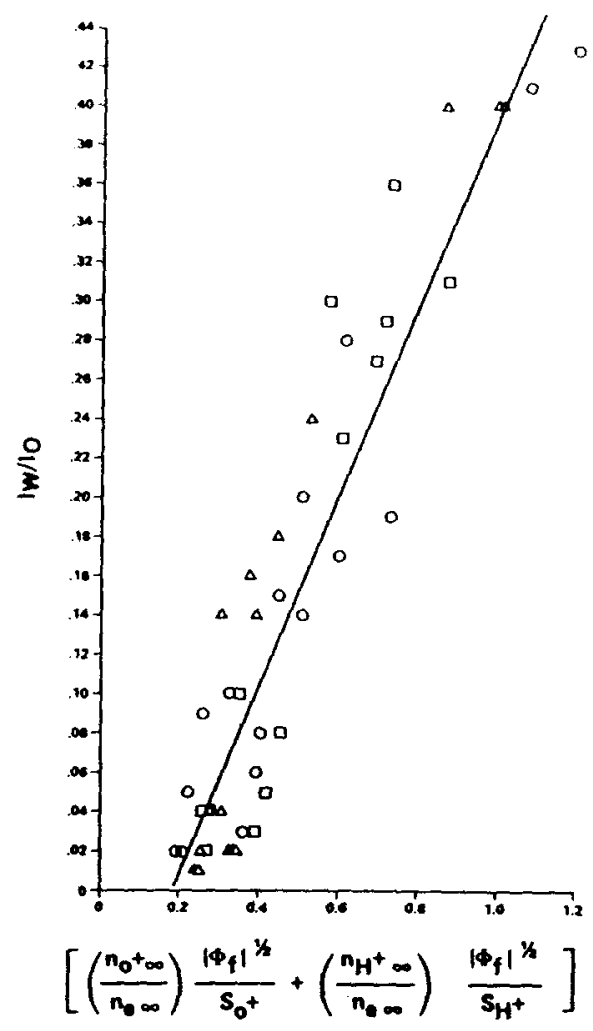

Fig. 7 Normalized ion current collected in the wake of the AE-C satellite as a function of $\left|\Phi_{f}\right| 1 / 2 / S$ for noninteracting ion constituents.

A schematic illustration of a possible method for conducting such experiments and several of the effects that may exdst are shown in Figure 8 . Note that this method allows simultaneous monitoring of the ambient plasma while the disturbed flow field created by the test body is mapped by a second, maneuverable set of diagnostic instruments.

\section{Application to Solar System Plasma Phenomena}

Within the region parameter space attainable in the ionosphere, there are cases where the characteristics of the plasma dynamic interaction with orbiting test bodies are qualitatively similar to those of certain natural interactions occurring in the solar system. This is particularly true of the interaction which occurs between planetary satellites and planetary magnetospheric plasmas. A number of such cases are known to exist at Jupiter and Saturn. The satellites involved cover a wide range of sizes and surface characteristics, and it is possible that some may include a dipole magnetic field. However, all are supersonic-subAlfvenic, which is the condition of orbiting test bodies in the Earth's ionosphere.

To clearly illustrate the quality of the scaling possible, we consider the specific case of the Jovian satellite Io. The best estimates of the plasma conditions and the resulting scaling parameters are shown in Table 1. For comparison, we have also shown the corresponding values for a $100 \mathrm{~m}$ test body in a $300 \mathrm{~km}$ circular orbit. 


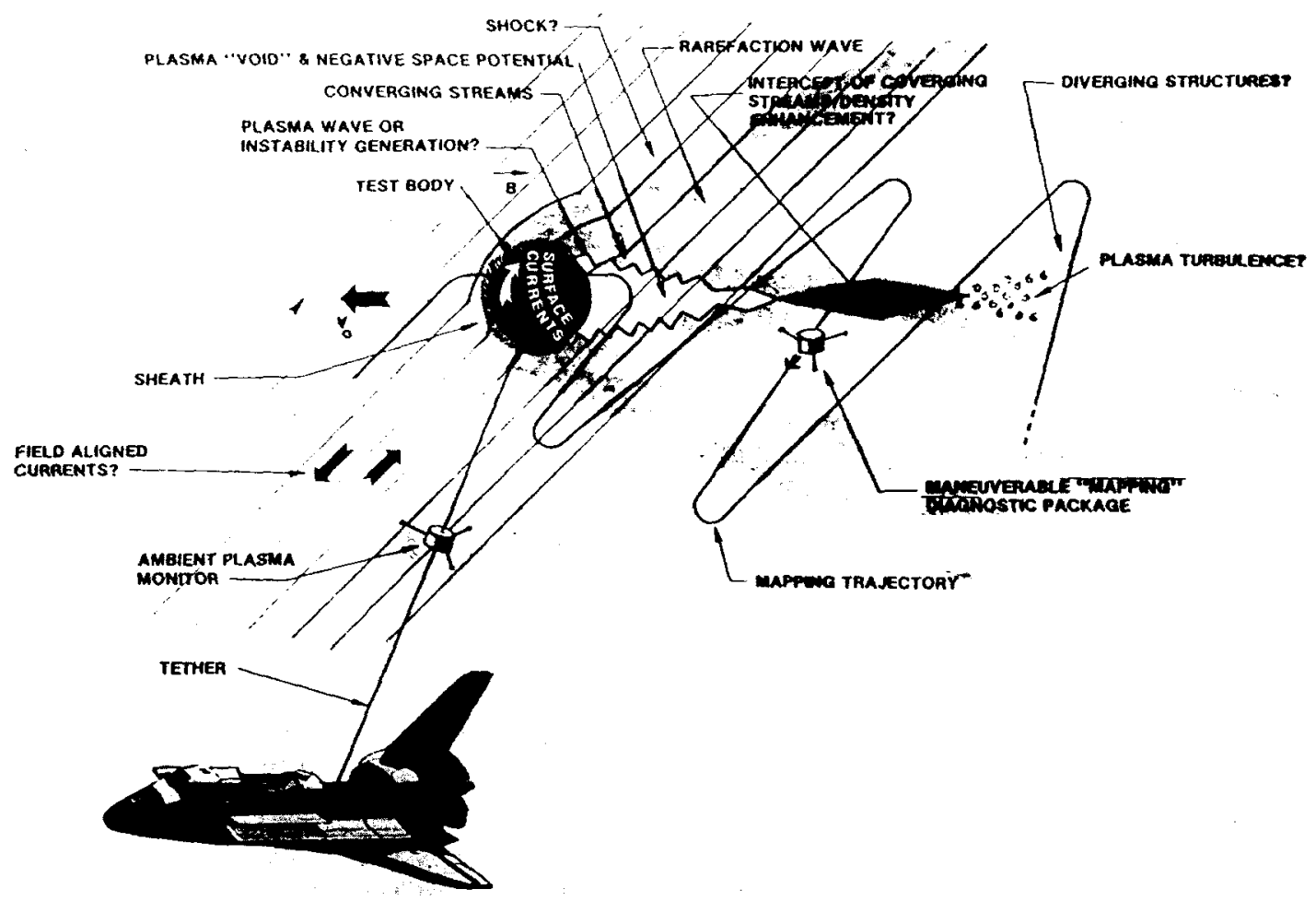

Fig. 8 Schematic of a large body plasma flow interaction study utilizing the Space Shuttle. Note that all effects shown would not be expected to occur under the same conditions and some may not exist.

For several parameters, the scaling is almost exact and, with the exception of the product of the ion cyclotron frequency and the characteristic time of flight, the values are well within the requirements of qualitative scaling.

It is anticipated that such experiments would supplement and elucidate the data obtained from planetary probes in much the same way as the results from laboratory studies have influenced the analysis of ionospheric satellite data. In fact, the degree of simulation between the conditions for Io and those of an ionospheric experiment is significantly better than that attained between the laboratory and ionospheric satellites. Yet, in spite of these limitations, we have shown the laboratory results to be pertinent to the satellite-ionospheric interaction.

\section{CONCLUSIONS}

We have endeavored to make essentially two points: First, in addition to reviewing some of the results from laboratory investigations of rarefied plasma flow interactions, we have shown, by example, how these results can be used to complement the fragmentary data currently available from ionospheric satellites that are applicable to the satellite-induced disturbance. Secondly, we have attempted to demonstrate the great potential of using these techniques to conduct qualitatively scaled experiments in the ionosphere by showing the excellent degree of scaling achievable for the interaction of lo with the Jovian magnetosphere. This is but one example of several phenomena of this type known to exist in the solar system. No doubt, additional uses of the ionospheric laboratory will surface once it begins to be utilized fully. 


\section{REFERENCES}

1. U. Samir, this volume.

2. K. Birkeland, in: The Norwegian Aurora Polaris Expedition, 1902-1903, Aschenhaug \& $\mathrm{Co}$. , Christiania, 1908.

3. L. Danielsson and L. Lindberg, Phys. Fluids 7, 1878 (1964).

4. L. Danielsson and L. Lindberg, Arkiv Fysik 28, 1 (1965).

5. J. W. Dungey, Phys. Rev. Letters 6, 47 (1961).

6. C. G. Falthamar, Space Sci. Rev. 15, 803 (1974).

7. E. M. Dubinin, I. M. Podgorny, Yu. M. Potanin, and C. P. Sonett, Geoph. Lett. 3, 391 (1977).

8. I. M. Podgorny and Yu V. Andrijanov, Planet. Space Sci. 26, 99 (1978).

9. I. M. Podgorny, this volume.

10. D. F. Hal1, R. F. Kemp, and J. M. Sellen, Jr., AIAA J. 3, 1490 (1965).

11. N. H. Stone and W. K. Rehmann, NASA TN-D-5894 (1970).

12. A. R. Martin and R. N. Cox, AIAA/NASA/ASTM/IES 7th Space Simulation Conference, Los Angeles, November 1973.

13. D. F. Hal1, R. F. Kemp, and J. M. Sellen, Jr., AIAA J. 2, 1032 (1964).

14. W. A. Clayden and C, V. Hurdle, Rarefied Gas Dynamics 2, 1717, (1966).

15. N. H. Stone, U. Samir, and K. H. Wright, Jr., J. Geophys. Res. 83, 1668 (1978).

16. U. Samir, N. H. Stone, and W. A. Oran, Astrophys. and Space Sci. 31, L1 (1974).

17. V. V. Skortsov and L. V. Nosachev, Cosmic Res. 6, 191 (1968).

18. V. V. Skortsov and L. V. Nosachev, Cosmic Res. 6, 718 (1968).

19. N. H. Stone, Ph.D. Dissertation, University of Alabama in Huntsville, (1979).

20. S. D. Hester and A. V. Sonin, Phys. Fluids 13, 641 (1970).

21. A. R. Martin, Planet. Space Sci. 22, 121 (1974).

22. N. H. Stone, Rev. Sci. Instrum. 48, 1458 (1977).

23. N. H. Stone, submitted to J. Plasma Phys. (1980).

24. G. Fournier and D. Pigache, Phys. Fluids 18, 1443 (1975).

25. J. C. Taylor, Planet. Space Sci. 15, 155 and 463 (1967).

26. A. V. Gurevich, L. P. Pitaevskii, and V. V. Smirnova, Space Sci. Rev. 9, 423 (1969).

27. G. Fournier, Publication No. 137, office Nat. d'Etudes et de Rescherches Aerospatiales, Paris (1971).

28. I. A. Bogashchenko, A. V. Gurevich, R. A. Salfmov, and Yu. I. Eide1'man, Soviet Phys. JETP 32, 841 (1971).

29. V. T. Astrelin, I, A. Bogashchenko, N. S. Buchel'nikova, and Yu. I. Eidel'man, Soviet Phys-Tech. Phys. 17, 1369 (1973).

30. C. L. Henderson and U. Samir, Planet. Space Sci. 15, 1499 (1967).

31. U. Samir, W. A. Oran, and N. H. Stone, Rarefied Gas Dynamics 2, D11.1 (1974).

32. U. Samir, L. H. Brace, and H. C. Brinton, Geophys. Res. Lett. 6, 101 (1979). 